\title{
Editorial Introduction: Logical Methods for Social Concepts
}

\author{
Andreas Herzig • Emiliano Lorini
}

Received: 28 March 2011 / Accepted: 29 March 2011 / Published online: 16 April 2011

(C) Springer Science+Business Media B.V. 2011

The present special issue follows the workshop Logical Methods for Social Concepts (LMSC'09) ${ }^{1}$ that we organised from 20th to 25th July 2009 during the European Summer School on Logic, Language and Information (ESSLLI'09) in Bordeaux. The aim of the workshop was to understand the potentialities and limitations of logical methods for the analysis of social concepts. Such concepts are central both in the social sciences (e.g. in economics, sociology, social psychology) and in some areas of computer science (multi-agent systems, distributed AI, social software). Examples are the concepts of power, cooperation, responsibility, delegation, trust, reputation, norm, convention, agreement, commitment, etc. More in detail, some fundamental questions that the workshop was intended to raise are the following:

- Is logic adequate to express social concepts in an accurate way?

- Are some of the basic assumptions in the existing logics for multi-agent systems (e.g. omniscience, perfect information about interaction structure, perfect recall) too strong to capture the fundamental aspects of social phenomena?

- Are some concepts relevant for social theory missing in these logics?

The 13 papers that were presented at the workshop covered not only the technical aspects of logics for multi-agent systems, but also multidisciplinary aspects from social sciences and a critical analysis of the existing logical frame-

${ }^{1} \mathrm{http} / / /$ www.irit.fr/ Andreas.Herzig/Esslli09

\footnotetext{
A. Herzig $(\bowtie) \cdot$ E. Lorini

University of Toulouse, IRIT, CNRS, 118 route de Narbonne,

31062 Toulouse Cedex 09, France

e-mail: herzig@irit.fr
} 
works for the specification of social concepts. The presentations stimulated lively discussions on various issues from different disciplinary perspectives (economics, social philosophy, logic for AI).

After the workshop we invited the authors of the LMSC'09 papers as well as the members of the program committee to submit revised and extended versions to a special issue of the Journal of Philosophical Logic. Each of the nine papers that were submitted was carefully evaluated by three reviewers, and four papers were selected for the special issue. They cover a broad area ranging from logics for game theory, social choice theory, and mechanism design, to logics for modelling normative concepts, individual attitudes, and group attitudes.

The paper "Reasoning about permitted announcements" by Philippe Balbiani and Pablo Seban presents a normative extension of Plaza's public announcement logic (PAL) [2] with dynamic modal operators for permission and for obligation. The logic the authors propose allows to express the concept of 'permission to say' which is of primary importance both for the analysis of communication in organisational contexts and for the specification of security protocols and policies.

The paper "Reasoning about Social Choice Functions" by Nicolas Troquard, Wiebe van der Hoek and Michael Wooldridge is focused on a very actual topic in the multi-agent domain, namely logics for social choice theory and mechanism design. They propose a logic for reasoning about about social choice functions integrating the concepts of strategic ability and agent preference, providing an axiomatisation and a decidability result. The also show that the logic can be used to decide whether a given social choice function is strategy-proof.

The paper "Making a Start with the stit-Logic Analysis of Intentional Action" by Jan Broersen proposes a logical analysis of individual intentionality that is couched in an extension of the group version of Belnap's STIT logic [1]. The latter is known as the logic of Seeing to it that, and its group version allows to express that a group of agents sees to it that some proposition is true. Its extension has modal operators of knowledge and intention; this allows to express that an agent performs a certain action intentionally and to distinguish intentional actions from non-intentional actions. Part of the paper focuses on the relationship between knowingly doing and intentionally doing.

Finally, the paper "Reasoning about collectively accepted group beliefs" by Raul Hakli and Sara Negri deals with the concept of collective acceptance, that can be viewed as a 'rival' of common belief. While common belief has been studied for decades, the concept of collective acceptance was introduced more recently in order to provide a theory of institutions that is grounded of collective attitudes [3]. The paper starts from the logic of that concept and proposes a proof-theoretic analysis.

We would like to thank the reviewers of the special issue whose work allowed to ensure a high quality issue. 


\section{References}

1. Belnap, N., Perloff, M., \& Xu, M. (2001). Facing the future: Agents and choices in our indeterminist world. New York: Oxford University Press.

2. Plaza, J. A. (1989). Logics of public communications. In M. Emrich, M. Pfeifer, M. Hadzikadic, \& Z. Ras (Eds.), Proceedings of the 4th international symposium on methodologies for intelligent systems (pp. 201-216).

3. Tuomela, R. (2002). The philosophy of social practices: A collective acceptance view. Cambridge: Cambridge University Press. 